\title{
Usefulness of Combined Measurement of Serum Soluble IL-2R and Angiotensin-Converting Enzyme in the Detection of Uveitis Associated with Japanese Sarcoidosis
}

This article was published in the following Dove Press journal:

Clinical Ophthalmology

Mami Ishihara

Akira Meguro (1D

Mizuho Ishido

Masaki Takeuchi

Etsuko Shibuya

Nobuhisa Mizuki

Department of Ophthalmology and Visual Science, Yokohama City University Graduate School of Medicine, Yokohama, Kanagawa 236-0004, Japan
Correspondence: Mami Ishihara;

Akira Meguro

Tel +8I-45-787-2683

Fax +8I-45-78I-9755

Email mamitaiga7@gmail.com;

akmeguro@yokohama-cu.ac.jp
Purpose: Angiotensin-converting enzyme (ACE) is conventionally used as a biomarker in the diagnosis of uveitis associated with sarcoidosis, but its sensitivity is relatively low. In this study, we investigated whether serum soluble interleukin-2 receptor (sIL-2R) is also useful as a diagnostic marker, in addition to ACE, in the detection of uveitis associated with sarcoidosis.

Patients and Methods: Data were analyzed from 126 patients with uveitis ( 52 sarcoidosis and 74 non-sarcoid uveitis) and 12 with primary intraocular lymphoma (PIOL) who had their serum sIL-2R and ACE levels measured.

Results: Serum sIL-2R level was elevated in $69.2 \%$ of patients with sarcoid uveitis, $5.4 \%$ of those with non-sarcoid uveitis, and $16.7 \%$ of those with PIOL. The sensitivity and specificity of an elevated sIL-2R level for the detection of sarcoidosis were $69.2 \%$ and $93.0 \%$, respectively. In contrast, serum ACE levels were elevated only in patients with sarcoid uveitis, with a sensitivity of $44.2 \%$ and specificity of $100 \%$. Furthermore, serum sIL-2R and/or ACE level was elevated in $75.0 \%$ of patients with sarcoid uveitis, which is higher than those who had elevated serum ACE level only $(44.2 \%, P=0.0025)$. The sensitivity and specificity of elevated sIL-2R and/or ACE in detecting sarcoid uveitis were $75.0 \%$ and 93.0\%, respectively. The PPV was 0.87 , and the NPV was 0.86 .

Conclusion: Compared with the sensitivity of serum ACE levels alone, combined measurement of both serum sIL-2R and ACE levels improves sensitivity in the detection of uveitis associated with sarcoidosis. Nevertheless, serum ACE alone remains useful thanks to its high specificity in the differentiation of uveitis patients, with sarcoidosis from those without sarcoidosis.

Keywords: sarcoid uveitis, soluble interleukin-2 receptors, sIL-2R, angiotensin-converting enzyme, ACE, primary intraocular lymphoma, PIOL

\section{Introduction}

Sarcoidosis is a cryptogenic, multisystem disease that is one of the major causes of uveitis. The diagnosis of sarcoidosis requires a compatible clinical picture, the histological demonstration of noncaseating granulomas, and the exclusion of other diseases capable of producing a similar clinical or histological picture. In addition to histological diagnosis, the International Workshop on Ocular Sarcoidosis (IWOS) has determined that a diagnosis of sarcoidosis in patients 
with suggestive ocular findings of sarcoidosis be supported by laboratory investigations, including an elevated level of serum angiotensin-converting enzyme (ACE), bilateral hilar lymphadenopathy (BHL), and other parameters. ${ }^{1}$ Concerning sarcoid uveitis, however, ACE levels are not always elevated, ${ }^{2-4}$ and one proposed diagnostic marker for sarcoidosis is serum soluble interleukin-2 receptor (sIL-2R). In particular, serum levels of sIL-2R are elevated in patients with active lung sarcoidosis, and sIL-2R is both a diagnostic marker and predictor of the severity of this condition. $^{5-9}$ A previous study showed that serum sIL-2R is superior to ACE in ruling out sarcoidosis in patients suspected of sarcoidosis. ${ }^{10}$ However, the diagnostic value of sIL-2R levels in sarcoid uveitis has not been well established. $^{2,11}$

Serum sIL-2R levels are also elevated in patients with nonHodgkin's B-cell malignant lymphoma. ${ }^{12}$ Measuring sIL-2R levels may help in the diagnosis of lymphoma, and monitoring the course of the disease. ${ }^{12,13}$ The histopathological manifestation of primary intraocular lymphoma (PIOL), also called primary vitreoretinal lymphoma, ${ }^{14}$ is non-Hodgkin's B-cell malignant lymphoma. ${ }^{14-16}$ Although PIOL appears to be distinguishable from uveitis by cytodiagnosis of vitreous biopsy samples, ${ }^{15}$ it may be misdiagnosed as uveitis, including sarcoidosis. $^{16}$ To date, the significance of the measurement of serum sIL-2R in patients with PIOL and the frequency of elevation of serum sIL-2R levels remain unknown.

In this study, we aimed to determine whether serum sIL-2R is also a useful diagnostic marker, either as a replacement for or in addition to ACE, the conventional marker in the detection of uveitis associated with sarcoidosis, as well as in the differentiation of sarcoid uveitis from non-sarcoid uveitis and PIOL.

\section{Patients and Methods}

\section{Patients}

We enrolled 126 patients with uveitis (52 patients with uveitis associated with sarcoidosis and 74 patients with non-sarcoid uveitis) and 12 patients with PIOL. All patients visited the Department of Ophthalmology, Yokohama City University Hospital from January 2012 to March 2016, and received outpatient care for more than one year. Blood samples were taken from all patients, in addition to other systemic examinations, at their first visit to our department to determine the underlying etiology of diseases. We excluded patients that had received systemic pre-treatment such as immunosuppressive medications and ACE inhibitors one month prior to the measurement of serum sIL-2R and ACE levels. All patients were adults aged older than 20 years. Data from the patients were analyzed retrospectively. Demographic characteristics of the study cohort are given in Tables 1 and 2 .

The patients with sarcoid uveitis comprised 13 males and 39 females with a mean age of $58.8 \pm 15.2$ years.

Table I Characteristics of Patients with Sarcoid Uveitis $(n=52)$

\begin{tabular}{|l|l|}
\hline Characteristic & n (Frequency) \\
\hline Gender, female & $39(75.0 \%)$ \\
Mean age ${ }^{a}$, years & $58.8 \pm 15.2$ \\
\hline Organ involvement & \\
Lung & $45(86.5 \%)$ \\
Skin & $10(19.2 \%)$ \\
Heart & $3(5.8 \%)$ \\
Kidney & $2(3.8 \%)$ \\
Parotid gland & $1(1.9 \%)$ \\
Liver & $1(1.9 \%)$ \\
\hline Uveitis & \\
Bilateral & $52(100.0 \%)$ \\
Anterior uveitis & $0(0.0 \%)$ \\
Intermediate uveitis & $4(7.7 \%)$ \\
Posterior uveitis & $0(0.0 \%)$ \\
Panuveitis & $48(92.3 \%)$ \\
\hline
\end{tabular}

Note: ${ }^{a}$ Data are presented as means \pm standard deviations.

Table 2 Diagnosis of Patients with Uveitis and Primary Intraocular Lymphoma

\begin{tabular}{|l|l|}
\hline Diagnosis & $\begin{array}{l}\text { Number of } \\
\text { Patients }\end{array}$ \\
\hline Sarcoid uveitis $^{\text {Definite }}{ }^{\mathrm{a}}$ & 52 \\
Presumed $^{\mathrm{a}}$ & $36(69.2 \%)$ \\
Non-sarcoid uveitis & $16(30.8 \%)$ \\
Vogt-Koyanagi-Harada disease $_{\text {Uveoscleritis }}$ & 74 \\
Behçet's disease & $2 \mathrm{I}(28.4 \%)$ \\
Acute anterior uveitis & $19(25.7 \%)$ \\
Posner-Schlossmann syndrome & $16(21.6 \%)$ \\
Tubulointerstitial nephritis and uveitis & $\mathrm{II}(14.9 \%)$ \\
syndrome & $\mathrm{I}(1.4 \%)$ \\
Crohn's disease & $\mathrm{I}(1.4 \%)$ \\
Ulcerative colitis & $\mathrm{I}(1.4 \%)$ \\
Reiter syndrome & $\mathrm{I}(1.4 \%)$ \\
Psoriasis & $\mathrm{I}(1.4 \%)$ \\
Juvenile idiopathic iridocyclitis & $\mathrm{I}(1.4 \%)$ \\
Primary intraocular lymphoma & $\mathrm{I}(\mathrm{I} .4 \%)$ \\
\hline
\end{tabular}

Note: ${ }^{a}$ According to the IWOS diagnostic criteria. 
A diagnosis of sarcoidosis was based on clinical findings and/or histological evidence of noncaseating epithelioid cell granulomas, after excluding other granulomatous diseases, at the Departments of Respiratory Medicine and/or Dermatology of Yokohama City University Hospital and affiliated hospitals. According to the IOWS criteria, ${ }^{1}$ the diagnosis of "definite" (biopsy-supported diagnosis) was made in 36 patients and "presumed" (compatible uveitis with the presence of BHL) was made in 16 patients. Fortyeight patients suffered from panuveitis and four from intermediate uveitis. Among 52 patients with sarcoid uveitis, lung lesions manifested in 45 patients (86.5\%), skin lesions in $10(19.2 \%)$, heart lesions in $3(5.8 \%)$, kidney lesions in $2(3.8 \%)$, parotid lesions in $1(1.9 \%)$, and liver lesions in $1(1.9 \%)$ (Table 1$)$. None of the patients showed neurologic, muscle, or arthritic lesions.

The patients with non-sarcoid uveitis consisted of 40 males and 34 females with a mean age of $49.6 \pm 16.5$ years. The diagnosis of patients enrolled in this study was shown in Table 2. We excluded patients with infectious uveitis and uveitis of unknown etiology, such as idiopathic granulomatous uveitis, from the non-sarcoid subgroup.

The patients with PIOL consisted of nine males and three females with a mean age of $62.9 \pm 13.8$ years. None had a history of immunosuppression or HIV infection. A diagnosis of PIOL was determined as follows. Vitreous humor samples were obtained by standard threeport pars plana vitrectomy. The samples were analyzed by cytology and/or molecular pathology. In addition, vitreal interleukin (IL)-10 and IL-6 cytokine levels were measured by enzyme-linked immunosorbent assay. An IL-10/ IL-6 ratio $>1$ in vitreous samples was considered as indirect evidence for the diagnosis and differentiation of PIOL from uveitis. ${ }^{15,17}$

The present study was performed following the Declaration of Helsinki. Approval for the study was obtained from the institutional review board of Yokohama City University Hospital, with the informed consent of all the participants implied by their return of the questionnaires before giving a blood sample.

\section{Measurements}

Serum sIL-2R was measured by a two-site chemiluminescent enzyme immunometric assay (SRL, Tokyo, Japan) and serum ACE by a colorimetric method using p-hydroxyhippuryl-1-histidyl-1-leucine (BML, Tokyo, Japan) as the substrate. According to the manufacturer, values higher than $543 \mathrm{U} / \mathrm{mL}$ for sIL-2R and $25 \mathrm{U} / \mathrm{L}$ for ACE showed levels that were elevated compared with that in the healthy population. Thus, levels above the cut-off values of 543 $\mathrm{U} / \mathrm{mL}$ for sIL-2R and $25 \mathrm{U} / \mathrm{L}$ for $\mathrm{ACE}$ were considered elevated.

\section{Statistical Analysis}

We assessed the normality of distribution of sIL-2R and ACE with the Kolmogorov-Smirnov test. ACE was normally distributed and sIL-2R showed a non-parametric distribution. The frequency of elevated levels of sIL-2R, ACE, and sIL-2R and/or ACE between patients with sarcoid uveitis and non-sarcoid uveitis, and between sarcoid uveitis and PIOL were statistically analyzed using Fisher's exact test. The frequency of elevated ACE levels was compared with that of elevated levels of sIL-2R and/or ACE in patients with sarcoid uveitis with Fisher's exact test. Furthermore, the clinical features of patients with and without elevated sIL-2R levels were compared with the Wilcoxon-Mann-Whitney test. Correlations between serum sIL-2R levels and serum ACE levels were determined using Spearman's rank-order correlation test. All statistical analyses were performed with EZR statistical software ( $\mathrm{R}$ version 3.2,2), and $P$ values $<0.05$ (twotailed) were regarded as indicating statistical significance.

The sensitivity, specificity, positive predictive value (PPV), negative predictive value (NPV), and positive likelihood ratio of the measurements were calculated as follows: sensitivity $=$ true positive/(true positive + false negative); specificity $=$ true negative/(true negative + false positive); $\mathrm{PPV}=$ true positive/(true positive + false positive); NPV $=$ true negative/(true negative + false positive); and positive likelihood ratio $=$ sensitivity $/(1-$ specificity $)$.

\section{Results}

Serum sIL-2R levels ranged 210-2250 U/mL (mean $\pm \mathrm{SD}$, $834.5 \pm 486.7 \mathrm{U} / \mathrm{mL}$ ) in patients with sarcoid uveitis, 204-1580 U/mL $(476.1 \pm 370.5 \mathrm{U} / \mathrm{mL})$ in patients with PIOL, and 168-945 U/mL (313.0 $\pm 127.7 \mathrm{U} / \mathrm{mL})$ in patients with non-sarcoid uveitis (Figure 1,Table 3). The frequency of elevated sIL-2R levels was $69.2 \%$ (36 of 52) in patients with sarcoid uveitis, $16.7 \%$ (2 of 12) in patients with PIOL, and 5.4\% (4 of 74) in patients with non-sarcoid uveitis (Table 3 ). The frequency of elevated sIL-2R levels was significantly higher among patients with sarcoid uveitis than among those with non-sarcoid uveitis $(P<0.0001)$ or PIOL $(P=0.002)$ (Table 3$)$. Patients with sarcoid uveitis had higher levels of serum sIL-2R $(834.5 \mathrm{U} / \mathrm{mL})$ 


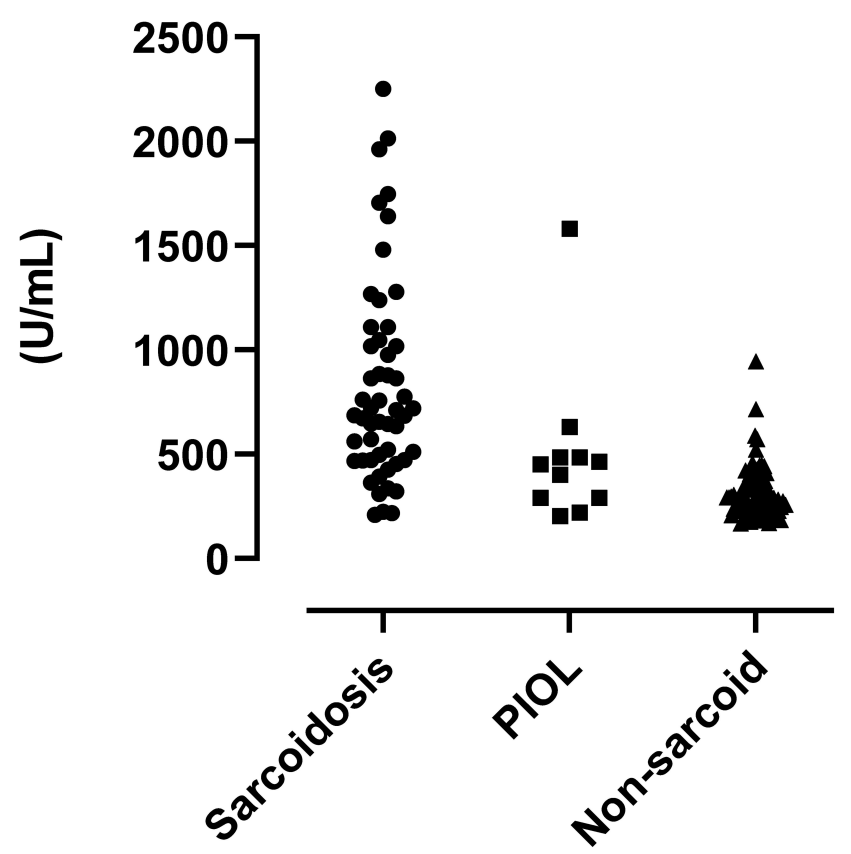

Figure I Comparison of sIL2R levels in patients with sarcoid uveitis, PIOL, and non-sarcoid uveitis. Serum sIL-2R levels ranged 210-2250 U/mL (mean, $834.5 \pm$ $486.7 \mathrm{U} / \mathrm{mL})$ in patients with 52 sarcoid uveitis, $204-1580 \mathrm{U} / \mathrm{mL}(476.1 \pm 370.5$ $\mathrm{U} / \mathrm{mL})$ in patients with $12 \mathrm{PIOL}$, and $168-945 \mathrm{U} / \mathrm{mL}(313.0 \pm 127.7 \mathrm{U} / \mathrm{mL})$ in patients with 74 non-sarcoid uveitis. Levels above the cut-off values of $543 \mathrm{U} / \mathrm{mL}$ were considered elevated.

than those with PIOL and non-sarcoid uveitis (476.1 and $313.0 \mathrm{U} / \mathrm{mL}$, respectively).

The sensitivity and specificity of an elevated sIL-2R level for the detection of sarcoidosis from cases with nonsarcoid uveitis and PIOL were 69.2\% and 93.0\%, respectively. The PPV was 0.86 , and the NPV was 0.83 . The positive likelihood ratio was 9.89 .

By contrast, serum ACE levels were elevated only in patients with sarcoid uveitis (range; 8.5-42.6 U/L, mean; $26.4 \pm 9.18 \mathrm{U} / \mathrm{L}$ ), with a frequency of $44.2 \%$ (23 of 52 patients) (Figure 2, Table 3), and a sensitivity of $44.2 \%$, specificity of $100 \%$. The PPV was 1.00 , and the NPV was 0.75 . Serum levels of ACE for PIOL and non-

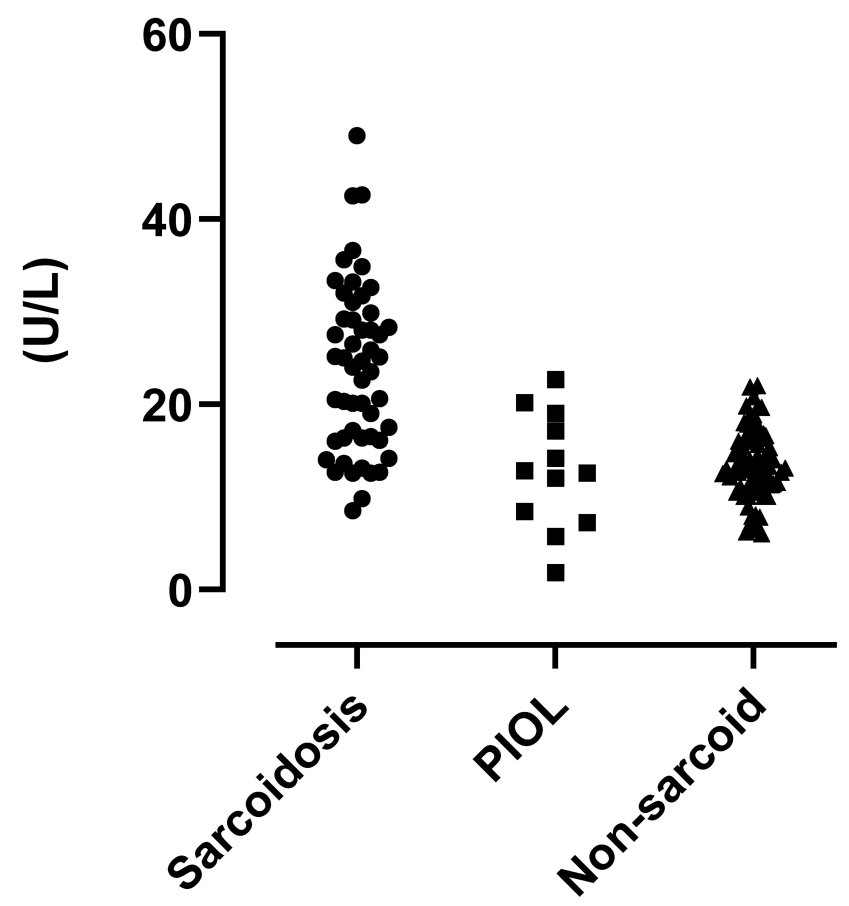

Figure 2 Comparison of ACE levels in patients with sarcoid uveitis, PIOL, and nonsarcoid uveitis. Serum ACE levels ranged 8.5-42.6 U/L (mean, $26.4 \pm 9.18 \mathrm{U} / \mathrm{L}$ ) in patients with 52 sarcoid uveitis, 5.7-22.7 U/L (12.8 $\pm 6.28 \mathrm{U} / \mathrm{L})$ in 12 patients with $\mathrm{PIOL}$, and 6.0-22.0 U/L ( $13.5 \pm 3.83 \mathrm{U} / \mathrm{L})$ in patients with 74 non-sarcoid uveitis. Levels above the cut-off values of $25 \mathrm{U} / \mathrm{L}$ were considered elevated.

sarcoid uveitis were $12.8 \pm 6.28 \mathrm{U} / \mathrm{L}$ (range; 5.7-22.7 U/L) and $13.5 \pm 3.83 \mathrm{U} / \mathrm{L}$ (range; 6.0-22.0 U/L), respectively (Figure 2, Table 3). The frequency of elevated serum ACE levels did not differ between patients under age 40, and patients aged 40 and over.

Furthermore, 39 of 52 patients with sarcoid uveitis $(75.0 \%)$ showed elevated levels of serum sIL-2R and/or ACE (Table 3). The frequency of these elevations was significantly higher among patients with sarcoid uveitis than among those with non-sarcoid uveitis $(P<0.0001)$ or PIOL $(P=0.0003)$ (Table 3$)$. In the patients with sarcoid uveitis, the frequency of elevated sIL-2R and/or ACE levels (75\%) was higher than that of elevated ACE

Table 3 Serum sIL-2R and ACE Levels in Patients with Sarcoid Uveitis, Primary Intraocular Lymphoma, and Non-Sarcoid Uveitis

\begin{tabular}{|c|c|c|c|c|c|}
\hline & \multicolumn{2}{|l|}{ sIL-2R } & \multicolumn{2}{|l|}{ ACE } & \multirow{2}{*}{$\begin{array}{l}\text { sIL-2R and/or ACE } \\
\text { Elevated No. (\%) }\end{array}$} \\
\hline & Mean \pm SD $(U / m L)$ & Elevated No. (\%) & Mean \pm SD (U/L) & Elevated No. (\%) & \\
\hline Sarcoid $(n=52)$ & $834.5 \pm 486.7$ & $36(69.2)$ & $26.4 \pm 9.18$ & $23(44.2)$ & $39(75.0)^{c}$ \\
\hline PIOL $(n=12)$ & $476.1 \pm 370.5$ & $2(16.7)^{a}$ & $12.8 \pm 6.28$ & $0(0.0)$ & $2(16.7)^{d}$ \\
\hline Non-sarcoid $(n=74)$ & $313.0 \pm 127.7$ & $4(5.4)^{\mathrm{b}}$ & $13.5 \pm 3.83$ & $0(0.0)$ & $4(5.4)^{e}$ \\
\hline
\end{tabular}

Notes: Levels above the cut-off values of $543 \mathrm{U} / \mathrm{mL}$ for sIL-2R, and of $25 \mathrm{U} / \mathrm{L}$ for ACE were considered elevated. $P$ value; calculated by Fisher's exact test. $P$ values $<0.05$ were considered significant. ${ }^{a} P=0.002$, sarcoid vs PIOL, ${ }^{b} P<0.0001$, sarcoid vs non-sarcoid, ${ }^{c} P=0.0025$, elevated ACE only vs elevated sIL-2R and/or ACE in sarcoid uveitis; ${ }^{\mathrm{d}} P=0.0003$, sarcoid uveitis vs $\mathrm{PIOL} ;{ }^{\mathrm{e}} \mathrm{P}<0.000 \mathrm{I}$, sarcoid uveitis vs non-sarcoid uveitis.

Abbreviations: Sarcoid, sarcoid uveitis; PIOL, primary intraocular lymphoma; non-sarcoid, non-sarcoid uveitis; SD, standard deviation. 

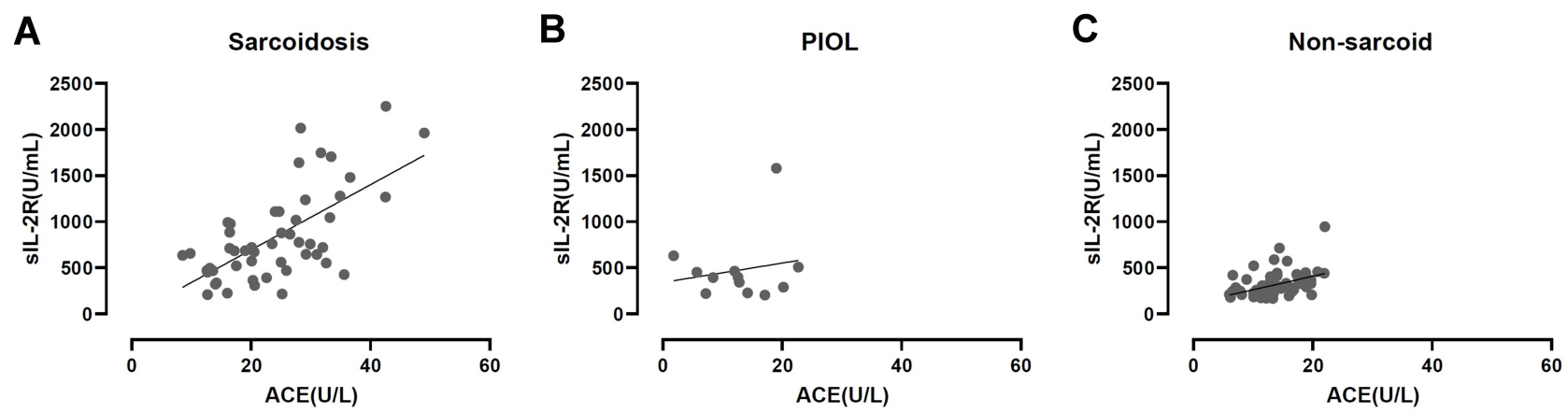

Figure 3 Correlation between serum sIL-2R and ACE levels in patients with sarcoid uveitis, PIOL, and non-sarcoid uveitis. (A); Sarcoid uveitis, (B); PIOL, (C); non-sarcoid uveitis. A Spearman Rank correlation analysis was performed to assess the relationship between serum slL-2R and ACE levels in each group. Positive correlation between two serum markers were observed in sarcoid uveitis $\left(\rho=0.595, P=3.18 \times 10^{-6}\right)$ and non-sarcoid uveitis $\left(\rho=0.492, P=8.56 \times 10^{-6}\right)$. There was no correlation between serum sIL-2R and ACE levels in PIOL $(\rho=-0.076, P=0.8 \mathrm{I})$.

levels only $(44.2 \%, P=0.0025)$ (Table 3$)$. The sensitivity and specificity of elevated sIL-2R and/or ACE for detecting sarcoid uveitis was $75.0 \%$ and $93.0 \%$, respectively. The PPV was 0.87 , and the NPV was 0.86 . The positive likelihood ratio was 10.71 . There were no statistically significant differences in the frequency of elevation of each marker or sIL-2R and/or ACE between definite and presumed ocular sarcoidosis $(P>0.05)$.

A total of 20 of $52(38.5 \%)$ sarcoid patients were double-positive for ACE and sIL-2R. Serum sIL-2R and ACE levels were positively correlated in patients with sarcoid uveitis $\left(\rho=0.595, P=3.18 \times 10^{-6}\right)$ (Figure 3A). There was no correlation between serum sIL-2R and ACE levels in the PIOL group ( $\rho=-0.076, P=0.81)$ (Figure 3B). Although the averages of both the serum sIL-2R and ACE levels were not elevated, a weak correlation was observed in patients with non-sarcoid uveitis $\left(\rho=0.492, P=8.56 \times 10^{-6}\right)$ (Figure 3C).

Furthermore, among patients with sarcoid uveitis, we investigated whether there were any clinical differences between those with and without elevated sIL-2R levels. Serum sIL-2R levels tended to be higher in patients who had lung lesions or had more than three organ lesions. However, the differences did not reach statistical significance $(P>0.05)$.

\section{Discussion}

This study investigated whether serum sIL-2R levels can be used as a diagnostic marker, in addition to serum ACE levels, in the detection of sarcoid uveitis and differentiation from non-sarcoid uveitis and PIOL. We found that serum sIL-2R levels for the detection of sarcoidosis had higher sensitivity $(69.2 \%$ vs $44.2 \%)$ but slightly lower specificity (93.0\% vs $100.0 \%)$ than serum ACE levels.
Meanwhile, the sensitivity and specificity of elevated sIL-2R and/or ACE were $75.0 \%$ and $93.0 \%$, respectively. Thus, our data suggest that the combined measurement of both serum sIL-2R and ACE levels improves sensitivity in the detection of uveitis associated with sarcoidosis. In contrast, serum ACE alone remains useful because of its high specificity in the differentiation of sarcoid uveitis from non-sarcoid uveitis and PIOL.

Serum sIL-2R, a proposed biomarker of sarcoidosis, is thought to be produced not only by activated $\mathrm{T}$ cells but also by alveolar macrophages. ${ }^{18}$ Measurement of sIL-2R as part of the routine diagnostic workup of patients with uveitis could help distinguish sarcoid uveitis from nonsarcoid uveitis because of the relatively high sensitivity (94\%) and specificity (98\%) of sIL-2R levels. ${ }^{2}$ Another study showed that the serum sIL-2R had $81 \%$ sensitivity and $64 \%$ specificity. ${ }^{11}$ These results are in agreement with this study, which found that the most probable diagnosis among uveitis cases was associated with sarcoidosis when serum sIL-2R levels were elevated. Also, patients with sarcoid uveitis had higher levels of serum sIL-2R (834.5 $\mathrm{U} / \mathrm{mL}$ ) than those with PIOL and non-sarcoid uveitis (476.1 and 313.0 U/mL, respectively) (Figure 1, Table 3).

ACE, the conventional biomarker of sarcoidosis, is produced by epithelioid cells of granulomas, and it is known that ACE concentrations may be related to the granuloma burden. ${ }^{19}$ Sensitivity of ACE in sarcoidosis were $62 \%$ in one study ${ }^{10}$ and $41.4 \%$ in a populationbased study. $^{20}$ In sarcoid uveitis, the sensitivity of ACE is relatively low, at $22 \%$ in a study by Gundlach et $\mathrm{al}^{2}$ and $55 \%$ in a study by Grajewski et al, ${ }^{3}$ as well as $44.2 \%$ in this study. The reason for this lower sensitivity of ACE compared to sIL2R may be because serum ACE levels are influenced by an ACE I/D polymorphism of the ACE 
gene. There was a difference in serum ACE in the three genotype groups, DD, ID, and II, showing inter-individual and ethnic variation. ${ }^{21,22}$ By contrast, the specificity of ACE levels in sarcoidosis was high, at $89.9 \%$ in a population-based study, and ACE elevation was associated with a 6-fold increase in the likelihood of sarcoidosis. $^{20}$ In addition, the specificity of serum ACE in sarcoid uveitis is extremely high, at $94 \%$ and $88 \%$ in previous reports, ${ }^{2,3}$ as well as $100 \%$ in this study. Therefore, ACE may still be a good marker for differentiating sarcoid uveitis from other uveitis because of its high specificity. On the other hand, more than half of our patients with sarcoid uveitis displayed normal ACE levels (Table 3), and only the measurement of serum ACE would be insufficient in detecting sarcoid uveitis.

The frequency of patients with sarcoid uveitis who had elevated serum levels of sIL-2R and/or ACE was higher than that of those who had only elevated serum ACE levels $(75.0 \%$ and $44.2 \%$, respectively; $P=0.0025)$ (Table 3). Although serum sIL-2R and ACE levels were positively correlated (Figure $3 \mathrm{~A}$ ), both biomarkers do not always exhibit parallel changes. Therefore, the combined measurement of both markers can improve sensitivity as compared to the measurement of serum ACE only. In this study, the PPV (0.87) and NPV (0.86) of elevated sIL-2R and/or ACE were reliable for the detection of sarcoid uveitis. In addition, we considered finding a new factor using these markers such as the sIL-2R/ACE ratio, but no other useful factor was found.

PIOL may masquerade as chronic posterior uveitis or panuveitis, with signs such as vitreous infiltration, retinal vasculitis, or multifocal chorioretinitis, ${ }^{14-16}$ sometimes resembling those of sarcoid uveitis. ${ }^{1}$ In this study, the proportion of patients with high levels of sIL-2R was significantly lower in patients with PIOL $(16.7 \%)$ than in patients with sarcoid uveitis $(69.2 \%)(P=0.002$, Table 3$)$. The median sIL-2R level was lower in patients with PIOL than in those with sarcoid uveitis $(476.1 \mathrm{U} / \mathrm{mL}$ vs $834.5 \mathrm{U} / \mathrm{mL})$ (Table 3 and Figure 1). There was no correlation between serum sIL-2R and ACE levels in patients with PIOL (Figure 3B). Furthermore, both sIL-2R and ACE did not correlate with the IL-10/IL-6 ratio in patients with PIOL (Supplemental Figure 1). In general, serum sIL-2R levels tend to be higher in patients with malignant lymphoma than in those with lung sarcoidosis. It was reported from Japan that sIL-2R levels ranged $195-3750 \mathrm{U} / \mathrm{mL}$ in patients with lung sarcoidosis and 240-62,300 U/mL in patients with nonHodgkin's lymphoma, and that sIL-2R levels in patients with lymphoma (stage III or IV) were significantly higher than those in patients with sarcoidosis. ${ }^{13}$ It is, therefore, possible that intraocular lesions in patients with PIOL are too small and limited to high serum sIL-2R levels, as compared to patients with sarcoid uveitis who have extraocular involvement, such as those in the present study. Since serum sIL-2R levels reflected overall disease activity or extent of disease in sarcoidosis, ${ }^{6}$ in this study, patients with lung lesions or with more than three organ lesions tended to have higher sIL-2R levels. Although the sIL-2R levels may not tend to increase in malignant lymphoma when lesions are restricted to the eyes, it is still difficult to distinguish sarcoid uveitis and PIOL simply from sIL-2R levels. Meanwhile, it is known that serum ACE levels tend to be within the normal range or lower in patients with malignant lymphoma, ${ }^{23}$ and none of our PIOL patients showed elevated ACE levels in this study. Therefore, serum ACE, thanks to its extremely high specificity, is still considered a good marker to distinguish sarcoidosis from PIOL. It is a fact that the necessity of cytodiagnosis of vitreous biopsy for differentiating PIOL from uveitis is established. Allowing that our results are preliminary and that the role of serum ACE and/or sIL-2R in differentiating between sarcoid uveitis and PIOL is limited, these conditions might initially warrant suspicion of sarcoidosis, especially in cases without lung lesions.

It is reported that chest radiography combined with serum sIL-2R or ACE improved specificity, as compared to the measurements of serum sIL-2R alone. ${ }^{11}$ In this study, $86.5 \%$ of the patients with sarcoid uveitis had lung lesions, and serum sIL-2R levels tended to be higher in patients who had lung lesions as compared to those without lung lesions. Although we focused on blood biomarkers in the current study, the diagnosis of sarcoidosis can be more supported by the presence of lung lesions, in addition to high levels of these markers.

The limitations of this study include the small number of patients in each subgroup and its retrospective, singlecenter design. Longitudinal measurement with a larger number of patients and multiple centers will be required to confirm our results.

In summary, this study demonstrates that while serum ACE remains a good marker in the differentiation of uveitis associated with sarcoidosis from non-sarcoid uveitis and PIOL because of its high specificity, the combined measurement of both serum sIL-2R and ACE levels offers higher sensitivity and might be more useful than the measurement of serum ACE levels alone in the detection of uveitis associated with sarcoidosis. 


\section{Acknowledgments}

We thank Drs Y. Hasumi, S. Kawano, and T. Yamane for assisting in examining the patients.

\section{Funding}

There is no funding to report.

\section{Disclosure}

The authors report no conflicts of interest. The authors alone are responsible for the content and writing of the paper.

\section{References}

1. Herbort CP, Rao NA, Mochizuki M; members of Scientific Committee of First International Workshop on Ocular Sarcoidosis. International criteria for the diagnosis of ocular sarcoidosis: results of the first International Workshop on Ocular Sarcoidosis (IWOS). Ocul Immunol Inflamm. 2009;17:160-169. doi:10.1080/09273940902818861

2. Gundlach E, Hoffmann MM, Prasse A, Heinzelmann S, Ness T, Rosenbaum JT. Interleukin-2 receptor and angiotensin-converting enzyme as markers for ocular sarcoidosis. PLoS One. 2016;11: e0147258. doi:10.1371/journal.pone.0147258

3. Grajewski RS, Adler W, Frank KF, et al. Predictive value of serum markers for pulmonary involvement in ocular sarcoidosis. Acta Ophthalmol. 2014;92:e250-251. doi:10.1111/aos.12248

4. Febvay C, Kodjikian L, Maucort-Boulch D, et al. Clinical features and diagnostic evaluation of 83 biopsy-proven sarcoid uveitis cases. $\mathrm{Br}$ J Ophthalmol. 2015;99:1372-1376. doi:10.1136/bjophthalmol-2014306353

5. Vorselaars AD, van Moorsel CH, Zanen P, et al. ACE and SIL-2R correlate with lung function improvement in sarcoidosis during methotrexate therapy. Respir Med. 2015;109:279-285. doi:10.1016/j. rmed.2014.11.009

6. Gungor S, Ozseker F, Yalcinsoy M, et al. Conventional markers in determination of activity of sarcoidosis. Int Immunopharmacol. 2015;25:174-179. doi:10.1016/j.intimp.2015.01.015

7. Zurkova M, Kolek V, Tomankova T, Kriegova E. Extrapulmonary involvement in patients with sarcoidosis and comparison of routine laboratory and clinical data to pulmonary involvement. Biomed Pap Med Fac Univ Palacky Olomouc Czech Repub. 2014;158:613-620. doi: $10.5507 /$ bp.2014.026

8. Miyoshi S, Hamada H, Kadowaki T, et al. Comparative evaluation of serum markers in pulmonary sarcoidosis. Chest. 2010;137:1391-1397. doi:10.1378/chest.09-1975

9. Bargagli E, Mazzi A, Rottoli P. Markers of inflammation in sarcoidosis: blood, urine, BAL, sputum, and exhaled gas. Clin Chest Med. 2008;29:445-458. doi:10.1016/j.ccm.2008.03.004
10. Eurelings LEM, Miedema JR, Dalm VASH, et al. Sensitivity and specificity of serum soluble interleukin-2 receptor for diagnosing sarcoidosis in a population of patients suspected of sarcoidosis. PLoS One. 2019;14:e0223897. doi:10.1371/journal.pone.0223897

11. Groen-Hakan F, Eurelings L, Ten Berge JC, et al. Diagnostic value of serum-soluble Interleukin 2 Receptor levels vs angiotensin-converting enzyme in patients with sarcoidosis-associated uveitis. JAMA Ophthalmol. 2017;135:1352-1358. doi:10.1001/jamaophthalmol.2017. 4771

12. Tangen JM, Abrahamsen AF, Ostenstad B, Bergheim J. Interleukin-2 receptor levels in non-Hodgkin's lymphoma: correlation to histological degree of malignancy and presence of constitutional symptoms. Acta Oncol. 1990;29:581-583. doi:10.3109/02841869009090055

13. Kita T, Watanabe S, Yano F, et al. Clinical significance of the serum IL-2R level and Ga-67 scan findings in making a differential diagnosis between sarcoidosis and non-Hodgkin's lymphoma. Ann Nucl Med. 2007;21:499-503. doi:10.1007/s12149-007-0060-9

14. Levasseur SD, Wittenberg LA, White VA. Vitreoretinal lymphoma: a 20-year review of incidence, clinical and cytologic features, treatment, and outcomes. JAMA Ophthalmol. 2013;131:50-55. doi:10.1001/jamaophthalmol.2013.569

15. Kimura K, Usui Y, Goto H; Japanese Intraocular Lymphoma Study Group. Clinical features and diagnostic significance of the intraocular fluid of 217 patients with intraocular lymphoma. Jpn J Ophthalmol. 2012;56:383-389. doi:10.1007/s10384-012-0150-7

16. Birnbaum AD, Huang W, Sahin O, Tessler HH, Goldstein DA. Ocular sarcoidosis misdiagnosed as primary intraocular lymphoma. Retina. 2010;30:310-316. doi:10.1097/IAE.0b013e3181b773fc

17. Wolf LA, Reed GF, Buggage RR, Nussenblatt RB, Chan CC. Vitreous cytokine levels. Ophthalmology. 2003;110:1671-1672. doi:10.1016/S0161-6420(03)00811-X

18. Ina Y, Takada K, Sato T, Yamamoto M, Noda M, Morishita M. Soluble interleukin-2 receptor in patients with sarcoidosis. Possible organ. Chest. 1992;102:1128-1133. doi:10.1378/chest.102.4.1128

19. Gilbert S, Steinbrech DS, Landas SK, Hunninghake GW. Amounts of angiotensin-converting enzyme mRNA reflect the burden of granulomas in granulomatous lung disease. Am Rev Respir Dis. 1993;148:483-486. doi:10.1164/ajrccm/148.2.483

20. Ungprasert P, Carmona EM, Crowson CS, Matteson EL. Diagnostic utility of angiotensin-converting enzyme in sarcoidosis: a population-based study. Lung. 2016;194:91-95. doi:10.1007/s00408015-9826-3

21. Furuya K, Yamaguchi E, Itoh A, et al. Deletion polymorphism in the angiotensin I converting enzyme (ACE) gene as a genetic risk factor for sarcoidosis. Thorax. 1996;51:777-780. doi:10.1136/thx.51.8.777

22. Fløe A, Hoffmann HJ, Nissen PH, Møller HJ, Hilberg O. Genotyping increases the yield of angiotensin-converting enzyme in sarcoidosis-a systematic review. Dan Med J. 2014;61:A4815.

23. Rømer FK, Emmertsen K. Serum angiotensin-converting enzyme in malignant lymphomas, leukemia and multiple myeloma. Br J Cancer. 1980;42:314-318. doi:10.1038/bjc.1980.232
Clinical Ophthalmology

\section{Publish your work in this journal}

Clinical Ophthalmology is an international, peer-reviewed journal covering all subspecialties within ophthalmology. Key topics include: Optometry; Visual science; Pharmacology and drug therapy in eye diseases; Basic Sciences; Primary and Secondary eye care; Patient Safety and Quality of Care Improvements. This journal is indexed on PubMed
Central and CAS, and is the official journal of The Society of Clinical Ophthalmology (SCO). The manuscript management system is completely online and includes a very quick and fair peer-review system, which is all easy to use. Visit http://www.dovepress.com/ testimonials.php to read real quotes from published authors. 